\title{
Respuestas morfológicas y anatómicas de Tessaria absinthioides (Hook. et Arn.) DC. a la salinidad ${ }^{1}$
}

\author{
CLAUDIA A. M. DEGANO²
}

(recebido em 10/06/97; aceito em 07/04/99)

\begin{abstract}
Morphology and anatomy of Tessaria absinthioides (Hook. et Arn.) DC. under salinity conditions).T. absinthioides, Inuleae, Compositae, is a weedy species that is spreading in the irrigation area of the Colorado River, Argentina. This species can be found in normal and saline soils. Morphological and anatomical variables were measured with two salts, sodium chloride and sodium sulfate, with three levels of osmotic potential $-0.4,-0.8$ and $-1.5 \mathrm{MPa}$, in hydroponic culture, using Hoagland solution as the cultivation media. The total diameter of the roots of plants growing in $\mathrm{Na}_{2} \mathrm{SO}_{4}$ and $\mathrm{NaCl}$ increased when the osmotic potential was $-0.4 \mathrm{MPa}$. In plants growing in $\mathrm{NaCl}$ this may have resulted from the increase in the size of the cortical cells and in plants growing in $\mathrm{Na}_{2} \mathrm{SO}_{4}$, the diameter increased may be due to an increase in the cambial activity. The number and length of shoot internodes decreased with increasing salinity, even though this was not statistically significant. In comparison to the control, the total diameter of the shoot increased at $-0.4 \mathrm{MPa}$ and decreased with the reduction of the osmotic potential. In comparison to the control, the length of the leaves decreased at $-0.4 \mathrm{MPa}$ and the leaves width increased at the same concentration. The palisade parenchyma appeared less developed in saline conditions. In comparison to the control, the number of hairs increased at $-0.4 \mathrm{MPa}$. T. absinthioides acts as a semihalophytic species, according to the salt ranks it tolerates. The mechanisms of adaptation to saline conditions are succulence in root and stunted growth if the salt in the media is $\mathrm{NaCl}$, and the production of haloxeromorphic characters if the salt in the media is $\mathrm{Na}_{2} \mathrm{SO}_{4}$
\end{abstract}

RESUMO - (Respostas morfológicas e anatômicas de Tessaria absinthioides (Hook. et Arn.) DC. à salinidade).Tabsinthioides, Inulae, Compositae, é uma espécie que se encontra difundida como erva daninha na área de irrigação do Rio Colorado, Argentina, tanto em solos salinos como normais. A fim de determinar a capacidade de adaptação da espécie a diferentes condições de salinidade, foram montados experimentos onde a planta foi submetida a dois tipos de sais, cloreto de sódio e sulfato de sódio, com três níveis de potencial osmótico: - $0,4,-0,8$ e -1,5 MPa, em cultura hidropônica, utilizando a solução de Hoagland como meio de cultura. O experimento foi avaliado com base nas variações quantitativas dos caracteres morfológicos e anatômicos. Os resultados mostram que o diâmetro das raízes aumenta quando o potencial osmótico dos sais é $-0,4 \mathrm{MPa}$; isto é devido ao aumento de tamanho das células da casca nas plantas submetidas ao $\mathrm{NaCl}$ ou ao aumento da atividade cambial nas colocadas em $\mathrm{Na}_{2} \mathrm{SO}_{4}$. No caule diminui o número e o comprimento dos entrenós, mas as diferenças observadas não são estatisticamente significativas. Tanto o diâmetro do caule, como o da raiz, aumentam quando o potencial osmótico atinge -0,4MPa, e diminuem com a redução do mesmo. Quando o potencial osmótico é $-0,4 \mathrm{MPa}$, a folha diminui de comprimento, aumenta sua largura e o número de pêlos; o parênquima paliçádico mostra-se menos desenvolvido em condições de salinidade. T. absinthioides mostra-se como semihalófita devido ao grau de tolerância à salinidade. $\mathrm{O}$ mecanismo de resposta das plantas ao $\mathrm{NaCl}$ é a produção de raízes suculentas e a redução do crescimento, enquanto que a presença de $\mathrm{Na}_{2} \mathrm{SO}_{4}$ produz caracteres haloxeromórficos.

Key words - Salinity, morpho-anatomical modifications, Tessaria

\section{Introducción}

Tessaria absinthioides se adapta a gran diversidad de condiciones edáficas, ya que vive en suelos de textura pesada a liviana, sometida tanto a elevadas como a bajas concentraciones salinas y a diversos regímenes de humedad, en costas marinas o en zonas mediterráneas. Debido a esta gran adaptabilidad y a su propagación asexual por medio de raíces gemíferas y rizomas, que asegura la

1. Parte de la tesis de Magister de C.A.M. Degano.

2. Facultad de Agronomía y Agroindustrias, Universidad $\mathrm{Na}$ cional de Santiago del Estero. Av. Belgrano (S) 1912. Código Postal 4200 Sgo. del Estero. Argentina. Fax: 54-3854214499. E-mail: claudeg@unse.edu.ar multiplicación de los genotipos de supervivencia comprobada en los distintos ambientes que es capaz de colonizar, se está extendiendo su área de dispersión en la zona de riego del Río Colorado (Argentina), constituyendo una maleza de regular importancia. Si bien varios autores (Chapman 1960, St.Omer \& Schlesinger 1980) citan a muchos géneros de la familia Compositae como halófitos, entre ellos Baccharis, Aster, Solidago, Jaumea y Pluchea, este último de la tribu Inuleae, ninguno de ellos menciona al género Tessaria. La única cita encontrada sobre la condición halófila de la especie en estudio es la de Uphof (1941), quien en una revisión sobre el tema menciona que conforma comunidades junto con especies de Baccharis y Atriplex en las costas del Pacífico de Sudamérica, 
especialmente Chile, donde constituye espesos arbustales. No ha podido encontrarse ningún registro sobre la relación entre el hábitat y la morfología de T. absinthioides. Las plantas objeto de este estudio crecen en un área de suelos arcillosos salinizados en Pradere junto con especies típicamente halófilas como Salicornia sp. y Distichlis sp.

T. absinthioides posee caracteres morfológicos, tales como moderada suculencia, estructura foliar isobilateral, presencia de pelos glandulares y gran cantidad de pelos no glandulares, que se citan como típicos de plantas halófilas (Purer 1936, Black 1958, Hansen et al. 1976, St.Omer \& Schlesinger 1980, Voronin et al. 1995). Esto llevó a preguntarse hasta qué punto dichos caracteres son respuestas adaptativas a las condiciones edáficas imperantes en la zona de estudio y a plantear la hipótesis que propone que $T$. absinthioides responde al hábitat salino mediante la aparición de suculencia en sus órganos vegetativos y mayor pubescencia en sus órganos aéreos. Los vegetales que viven en condiciones de salinidad disponen de diversos mecanismos con los que pueden regular y mantener la concentración interna de iones. Algunos de ellos son: glándulas de sal, parénquima acuífero, abscisión de partes del vegetal, achaparramiento y presencia de caracteres haloxeromórficos (Strogonov 1964, Waisel 1972, Poljakoff-Mayber \& Gale 1975, Jennings 1976, Huang \& Redmann 1995a,b). Como T. absinthioides no posee glándulas de sal como mecanismo de adaptación, otros caracteres cumplirían tal función. El objetivo de este trabajo es detectar las modificaciones morfológicas y anatómicas que se operan en esta especie y que posibilitan su supervivencia en suelos de alta concentración salina como ocurre con las poblaciones de Pradere seleccionadas para este estudio.

\section{Materiales y metodos}

Del área de estudio, Pradere (62³7' W 39 $23^{\circ}$ S S), Provincia de Buenos Aires, Argentina, se determinó la salinidad del suelo, determinándose la presencia de 175,1 meq/1, 238,2 meq/1 y 8,5 $\mathrm{meq} / \mathrm{l}$ de los aniones cloruro, sulfato y carbonato, respectivamente; los cationes presentes en dicho suelo fueron sodio (294,0 meq/1), potasio $(3,7 \mathrm{meq} / 1)$, calcio $(80,0 \mathrm{meq} / \mathrm{l})$ y magnesio $(44,1 \mathrm{meq} / \mathrm{l})$. Se extrajeron raíces de diámetro semejante y se cortaron trozos de $10 \mathrm{~cm}$ de longitud utilizándolos como medio de regeneración, los que de aquí en más se llamarán trozos madre.

Se ensayaron tres concentraciones de cloruro de sodio $(\mathrm{NaCl})$ y tres de sulfato de sodio $\left(\mathrm{Na}_{2} \mathrm{SO}_{4}\right)$ cuyos rangos de potencial osmótico y molaridad se indican en la tabla 1 . Se asignaron a cada tratamiento 30 trozos madre elegidos al azar. Los mismos se
Tabla 1. Tratamientos aplicados, con la molaridad de cada sal y el potencial osmótico de cada solución.

\begin{tabular}{cccc}
\hline Tratamiento & Sal & $\begin{array}{c}\text { Concentración } \\
(\mathrm{M})\end{array}$ & $\begin{array}{c}\text { Potencial Osmótico } \\
(\mathrm{MPa})\end{array}$ \\
\hline$-0,4$ & $\mathrm{NaCl}$ & 0,041 & $-0,4$ \\
$-0,8$ & $\mathrm{NaCl}$ & 0,094 & $-0,8$ \\
$-1,5$ & $\mathrm{NaCl}$ & 0,200 & $-1,5$ \\
$-0,4$ & $\mathrm{Na}_{2} \mathrm{SO}_{4}$ & 0,031 & $-0,4$ \\
$-0,8$ & $\mathrm{Na}_{2} \mathrm{SO}_{4}$ & 0,069 & $-0,8$ \\
$-1,5$ & $\mathrm{Na}_{2} \mathrm{SO}_{4}$ & 0,115 & $-1,5$ \\
\hline
\end{tabular}

distribuyeron en seis recipientes de plástico con tapa, de un litro de capacidad. Cada trozo madre se ubicó en posición vertical sostenido por cortes realizados en cruz en la tapa y con un $70 \%$ de su longitud sumergida en la solución. Se utilizó como medio de cultivo solución Hoagland modificada (Hoagland \& Arnon 1950). Para lograr el potencial osmótico deseado y evitar el shock salino, las sales se agregaron progresivamente durante cuatro días y el quinto se consideró como el día inicial de aplicación de los tratamientos. Las soluciones se cambiaron semanalmente para mantener constantes las concentraciones. Como control se utilizaron otros treinta trozos madre repartidos en seis recipientes con solución de Hoagland modificada $(-0,005 \mathrm{MPa})$

La experiencia se llevó a cabo en invernáculo durante sesenta días. Los trozos madre comenzaron la producción de rebrotes y raíces al séptimo día. Sobre dichos brotes y raíces nuevos se realizaron las mediciones, descartando para tal fin los trozos madre. Para evaluar el crecimiento se midieron los siguientes parámetros morfológicos: diámetro de raíz y tallo, número y longitud de los entrenudos y largo y ancho foliar. Los datos se tomaron a los treinta días de aplicados los tratamientos. A los sesenta días se tomaron las muestras para los estudios anatómicos. En el tratamiento $-1,5 \mathrm{Cl}^{-}$se produjo la muerte de todos los rebrotes, razón por la cual no se registran datos para el mismo. Se midió en raíz y tallo el espesor de la corteza y médula, el tamaño de las células parenquimáticas de estas zonas, tamaño de las lagunas de la corteza y número de ellas por campo (un campo microscópico $=0,302 \mathrm{~mm}^{2}$ ) y el diámetro interno de los vasos. En las hojas se midió, en corte transversal, el espesor total en la vena media, el espesor del parénquima en empalizada y del parénquima incoloro en la parte media del semilimbo y el número de pelos en esta misma zona en una sección de epidermis de 0,62 mm de longitud. En cada caso se eligió la hoja superior madura y el primer entrenudo entre nomofilos para realizar los estudios anatómicos. Las mediciones anatómicas se realizaron sobre material fijado en FAA, deshidratado y infiltrado en parafina, cortado en secciones de $15 \mathrm{~mm}$ y teñido con safranina-fast green. Se realizó el estudio estadístico de los datos usando análisis de la varianza, las diferencias entre los tratamientos se estudiaron por la prueba de SNK (Statgraphics 1992)

\section{Resultados}

Los resultados muestran diferencias en el comportamiento entre las plantas sometidas al cloruro de sodio y al sulfato de sodio, las que se manifiestan por la diferente capacidad de regeneración de los trozos de raíces y de supervivencia de los brotes (tabla 2). 
Tabla 2. Número de brotes a los 40 y 65 días del ensayo, porcentaje de brotación y supervivencia, para cada tratamiento y el testigo.

\begin{tabular}{cccccccc}
\hline & $-0,4 \mathrm{Cl}^{-}$ & $-0,8 \mathrm{Cl}^{-}$ & $-1,5 \mathrm{Cl}^{-}$ & $-0,4 \mathrm{SO}_{4}{ }^{-}$ & $-0,8 \mathrm{SO}_{4}^{=}$ & $-1,5 \mathrm{SO}_{4}^{=}$ & $\mathrm{Testigo}^{=}$ \\
\hline 40 días & 15 & 6 & 5 & 21 & 12 & 0 & 28 \\
65 días & 5 & 4 & 0 & 7 & 3 & 0 & 21 \\
Brot. (\%) & 50 & 20 & 16 & 70 & 40 & 33 & 93 \\
Superv. (\%) & 33 & 66 & 0 & 33 & 25 & 0 & 75 \\
\hline
\end{tabular}

Raíz - El diámetro total de las raíces de plantas sometidas tanto al $\mathrm{Na}_{2} \mathrm{SO}_{4}$ como al $\mathrm{NaCl}$ muestra un incremento respecto al testigo en el potencial $-0,4$ (figura 1A), para disminuir con el aumento de la concentración. Esta variación del diámetro total es debida, en el caso de las plantas sometidas al cloruro de sodio, a un incremento notable del espesor de la corteza (figura 1B), causada por el aumento de tamaño de las células (tabla 3 ) que la forman. No se detectó aumento del número de capas de la corteza.

En las plantas sometidas al $\mathrm{Na}_{2} \mathrm{SO}_{4}$ el espesor de la corteza muestra una tendencia decreciente respecto al testigo aunque la reducción no fue significativa (figura 1B) y poca diferencia en el tamaño de las células que la componen; por lo tanto ninguna de estas variables, espesor de la corteza y tamaño de las células corticales, explica el aumento del diámetro total de las raíces en el tratamiento - 0,4 $\mathrm{SO}_{4}=$ lo que se debió quizás a una mayor actividad cambial. Se observó una tendencia creciente del número de lagunas que conforman el aerénquima cortical (figura 1C) con la disminución del

Tabla 3. Variaciones de los parámetros anatómicos para los diferentes tratamientos

\begin{tabular}{lccccc}
\hline & $\begin{array}{c}\text { Tamaño de } \\
\text { las células } \\
\text { de la corteza } \\
\text { de la raíz } \\
(\mu \mathrm{m})+\end{array}$ & $\begin{array}{c}\text { Diámetro } \\
\text { interno de } \\
\text { los vasos } \\
\text { en raíz } \\
(\mu \mathrm{m})^{*}\end{array}$ & $\begin{array}{c}\text { Diámetro } \\
\text { interno de } \\
\text { los vasos } \\
\text { del tallo } \\
(\mu \mathrm{m})^{*}\end{array}$ & $\begin{array}{c}\text { Ancho } \\
\text { foliar } \\
(\mathrm{mm})^{*}\end{array}$ & $\begin{array}{c}\text { Pelos. } \\
\mathrm{mm}^{-1 *}\end{array}$ \\
\hline Testigo $^{-4}$ & $40,1^{\mathrm{a}}$ & $40,6^{\mathrm{b}}$ & $24,1^{\mathrm{a}}$ & $4,4^{\mathrm{a}}$ & $7,5^{\mathrm{a}}$ \\
$-0,4 \mathrm{SO}_{4}{ }^{=}$ & $42,5^{\mathrm{ab}}$ & $35,1^{\mathrm{c}}$ & $28,4^{\mathrm{b}}$ & $4,3^{\mathrm{a}}$ & $11,8^{\mathrm{b}}$ \\
$-0,8 \mathrm{SO}_{4}{ }^{-}$ & $47,3^{\mathrm{ab}}$ & $24,1^{\mathrm{a}}$ & $10,9^{\mathrm{a}}$ & $2,4^{\mathrm{a}}$ & - \\
$-1,5 \mathrm{SO}_{4}{ }^{-}$ & $46,1^{\mathrm{ab}}$ & $14,7^{\mathrm{d}}$ & $14,7^{\mathrm{a}}$ & $2,7^{\mathrm{a}}$ & - \\
$-0,4 \mathrm{Cl}^{-}$ & $55,8^{\mathrm{b}}$ & $24,1^{\mathrm{a}}$ & $27,2^{\mathrm{b}}$ & $3,5^{\mathrm{a}}$ & $14,9^{\mathrm{c}}$ \\
$-0,8 \mathrm{Cl}^{-}$ & $54,6^{\mathrm{b}}$ & $26,0^{\mathrm{a}}$ & $11,9^{\mathrm{b}}$ & $3,3^{\mathrm{a}}$ & - \\
\hline
\end{tabular}

+ Promedios seguidos por letras distintas indican diferencias significativas a $\mathrm{p}=0,05$ con la prueba de $\mathrm{SNK}$.

* Los promedios seguidos de letras distintas son significativamente diferentes $(\mathrm{p}=0,01)$ usando la prueba SNK.
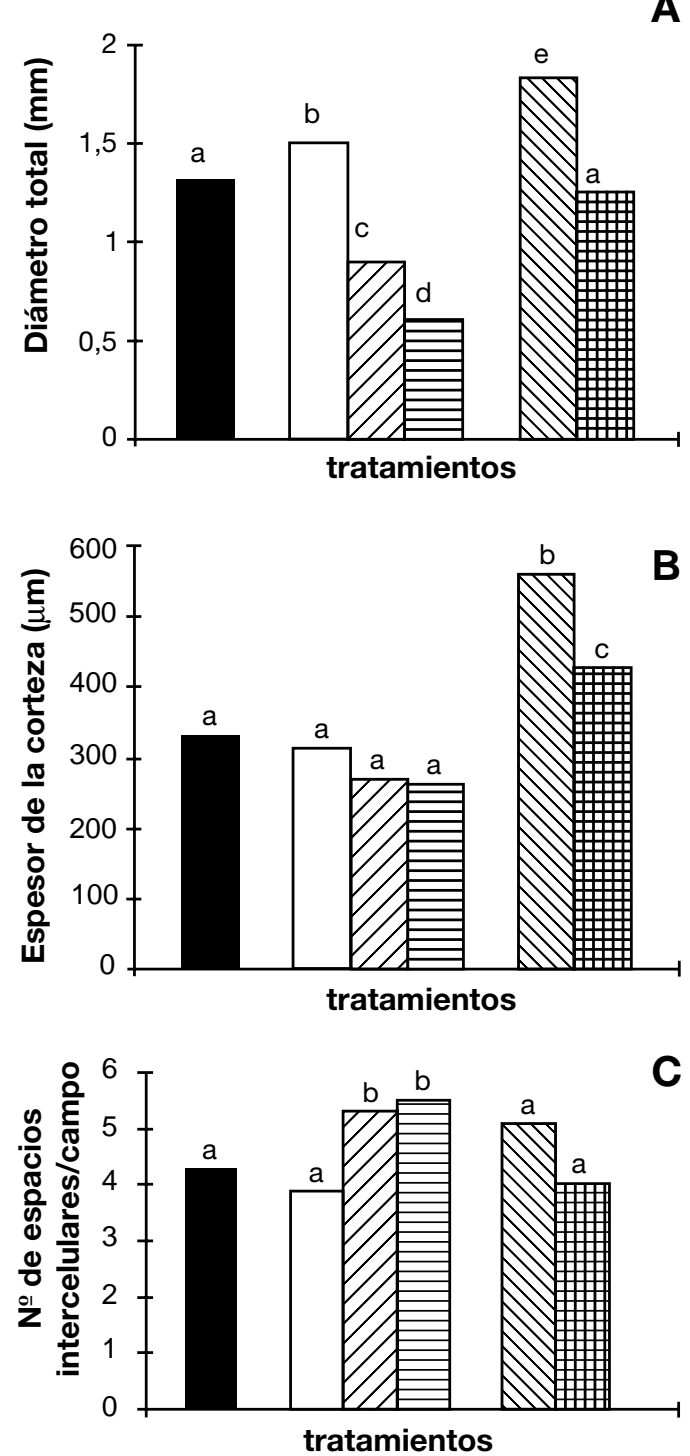

Figura 1. Variación del diámetro total de la raíz en función de las diferentes concentraciones y sales (A), variación del espesor de la corteza (B) y número de espacios intercelulares em la corteza de la raíz (C). testigo; $\square \mathrm{Na}_{2} \mathrm{SO}_{4}-0,4 \mathrm{MPa} ; \square \mathrm{Na}_{2} \mathrm{SO}_{4}-0,8 \mathrm{MPa}$; 目 $\mathrm{Na}_{2} \mathrm{SO}_{4}-1,5 \mathrm{MPa} ; \mathrm{NaCl}-0,4 \mathrm{MPa}$; NaCl-0,8MPa. 
potencial osmótico de $-0,4 \mathrm{SO}_{4}=\mathrm{a}-1,5 \mathrm{SO}_{4}{ }^{=}$manifestando el comportamiento inverso en los tratamientos $-0,4 \mathrm{Cl}^{-}$y $-0,8 \mathrm{Cl}^{-}$. El diámetro interno de los vasos disminuye respecto al testigo al someter a las plantas a un ambiente salino; esto se observa claramente en plantas expuestas a las distintas concentraciones de $\mathrm{Na}_{2} \mathrm{SO}_{4}$ (tabla 3) mientras que en las plantas sometidas al $\mathrm{NaCl}$, la variación en el diámetro interno de los vasos no es significativamente diferente (tabla 3 ).

Tallo - Se observa una tendencia decreciente en el número (figura 2A) y la longitud (figura 2B) de los entrenudos de las plantas sometidas tanto al $\mathrm{Na}_{2} \mathrm{SO}_{4}$ como al $\mathrm{NaCl}$, pero no se manifiestan diferencias significativas entre las distintas concentraciones excepto en $-0,8 \mathrm{Cl}^{-}$en que la longitud de los
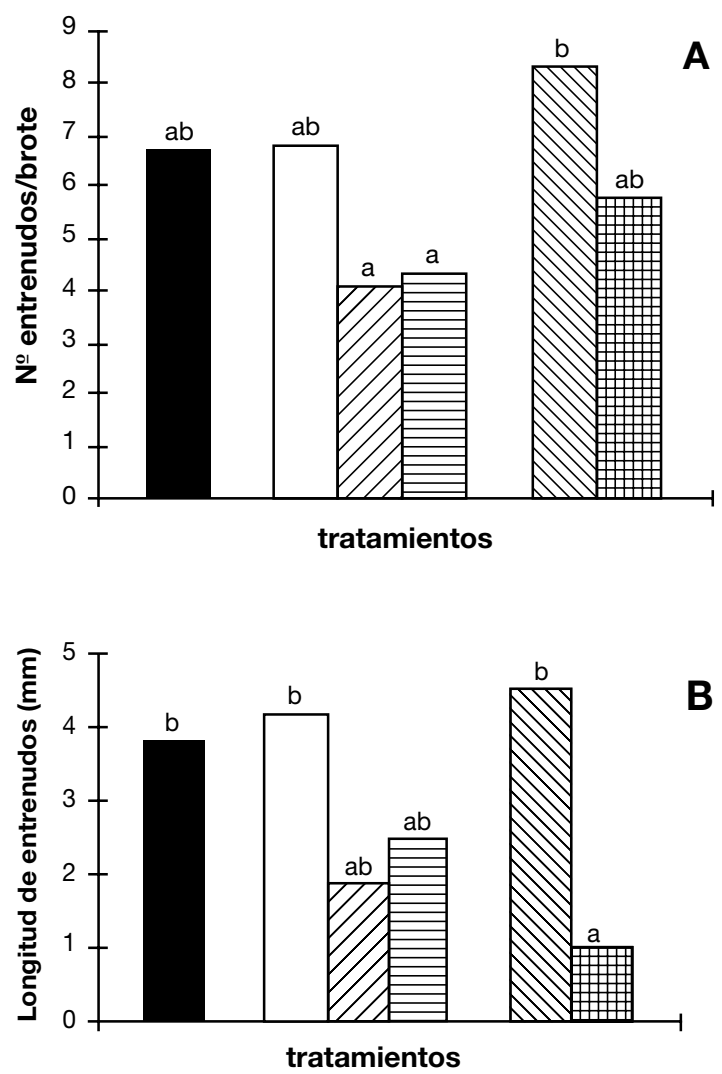

Figura 2. Efectos del medio salino en el número de entrenudos (A) y la longitud de los entrenudos (B) de los vástagos. $\square$ testigo; $\square \mathrm{Na}_{2} \mathrm{SO}_{4}-0,4 \mathrm{MPa} ; \square \mathrm{Na}_{2} \mathrm{SO}_{4}-0,8 \mathrm{MPa}$; 目 $\mathrm{Na}_{2} \mathrm{SO}_{4}-1,5 \mathrm{MPa}$; $\mathbb{N a C l}-0,4 \mathrm{MPa} ; \mathrm{NaCl}-0,8 \mathrm{MPa}$. entrenudos es menor, adoptando estos rebrotes apariencia achaparrada. El diámetro total del tallo (figura $3 \mathrm{~A}$ ) es mayor en los tratamientos $-0,4 \mathrm{SO}_{4}=$ y $-0,4 \mathrm{Cl}^{-}$, disminuyendo casi bruscamente con la reducción del potencial osmótico. Las variaciones del diámetro total podrían deberse, en las plantas sometidas al $\mathrm{NaCl}$, a un mayor diámetro de la médula y no a un mayor espesor de la corteza como se aprecia en la figura $3 \mathrm{~B}$. En $-0,8 \mathrm{Cl}^{-}$el diámetro de la médula (figura $3 \mathrm{~B}$ ) disminuye tanto respecto al tratamiento $-0,4 \mathrm{Cl}^{-}$como al testigo, resultando en un menor diámetro total. Cuando las plantas se encuentran en el medio salinizado con $-0,4 \mathrm{SO}_{4}=\mathrm{el}$ aumento del diámetro total es producto de las variaciones conjuntas del diámetro de la médula y del espesor de la corteza (figura 3B). El diámetro interno de los vasos se reduce (tabla 3 ) al disminuír el potencial osmótico de - $0,4 \mathrm{a}-0,8 \mathrm{MPa}$, tanto en las soluciones con $\mathrm{Na}_{2} \mathrm{SO}_{4}$, como en las con $\mathrm{NaCl}$ aunque se observa un incremento, respecto al testigo, en la menor concentración salina tanto de sulfato como de cloruro.

Hoja - La longitud de la hoja (figura 4A) muestra tendencia decreciente con la disminución del potencial osmótico, la que es menor que en las plantas control. El ancho de la hoja no muestra variaciones estadísticamente significativas pero hay una tendencia a su disminución con el aumento de la concentración respecto al testigo (tabla 3 ). Se manifiesta en $-0,4 \mathrm{SO}_{4}{ }^{=}$y $-0,4 \mathrm{Cl}^{-}$un aumento respecto al testigo, del espesor total de la hoja (figura 4B) mientras que el parénquima en empalizada tiene menor espesor que en el testigo (figura 4B). Entre las distintas presiones osmóticas no se detectan B diferencias estadísticamente significativas, excepto a $-1,5 \mathrm{SO}_{4}{ }^{=}$en que la hoja recién comenzaba a diferenciar su parénquima en empalizada, por lo que no se midió tal parámetro. Ante un potencial osmótico de - 0,4 MPa, debido tanto a la presencia de sulfato como de cloruro de sodio en el medio, se produjeron hojas más pubescentes (tabla 3 ) que en el testigo. La disminución de la longitud foliar en relación al número de pelos/mm, daría la pauta de que no se suspendió la división celular, sino que se produjo una reducción del tamaño de las mismas, puesto que en hojas más cortas encontramos más pelos por unidad de longitud. En el testigo se contaron 184,5 pelos/mm aproximadamente, en $-0,4 \mathrm{SO}_{4}=216,5$ pelos $/ \mathrm{mm}$ y en $-0,4 \mathrm{Cl}^{-} 233,9$ pelos $/ \mathrm{mm}$. 

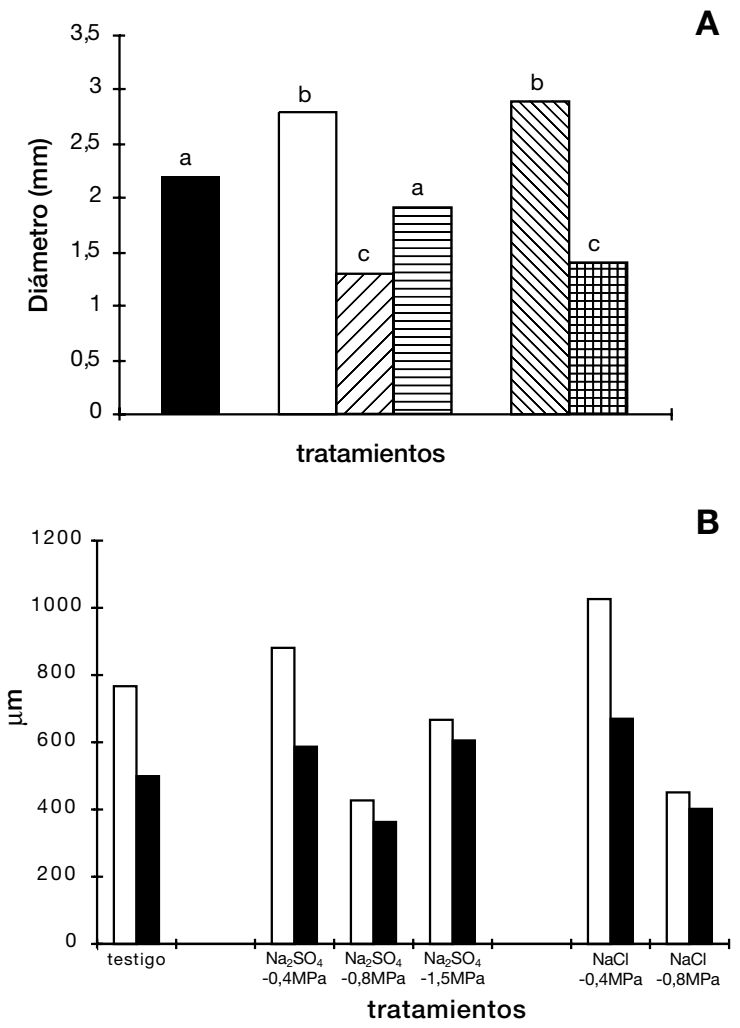

Figura 3. Efecto del medio salino: A. en el diámetro total testigo; $\square \mathrm{Na}_{2} \mathrm{SO}_{4}-0,4 \mathrm{MPa} ; \square \mathrm{Na}_{2} \mathrm{SO}_{4}-0,8 \mathrm{MPa}$; $\mathrm{Na}_{2} \mathrm{SO}_{4}$ -1,5MPa; $\mathrm{NaCl}-0,4 \mathrm{MPa}$; $\mathrm{NaCl}-0,8 \mathrm{MPa}$ ); B. en el diámetro de la médula $(\square)$ y espesor de la corteza del tallo

\section{Discusión}

Las plantas de T. absinthioides sometidas a los distintos tratamientos de salinidad se muestran afectadas en distinto grado. En las raíces de las plantas sometidas al $\mathrm{NaCl}$ se produce suculencia por incremento del tamaño de las células parenquimáticas de la corteza y no por el aumento del número de capas, coincidiendo con lo observado en otras especies sometidas a condiciones semejantes de salinidad (Black 1958, Waisel 1972). La suculencia es el efecto típico manifestado por plantas sometidas al cloruro de sodio (Strogonov 1964, Black 1958, Jennings 1976). Jennings (1968, 1976) sugiere como hipótesis que el aumento de suculencia de los distintos órganos de plantas
A halófilas, es una consecuencia de la diferencia de presión osmótica entre la planta y el medio externo, lo que produce un aumento de la turgencia de las células con el consiguiente aumento de tamaño y considera que beneficia a las plantas que viven en condiciones salinas, ya que el alto contenido de agua que se acumula en las células parenquimáticas tiene efecto de dilución del contenido iónico de tales células.

La presencia de aerénquima en la raíz observada en plantas de T. absinthioides sometidas al tratamiento con $\mathrm{Na}_{2} \mathrm{SO}_{4}$, podría contribuír a mejorar

el intercambio gaseoso, facilitando el proceso respiratorio. Hansen et al. (1976) observaron esta característica en plantas de Distichlis que crecieron en suelos saturados.
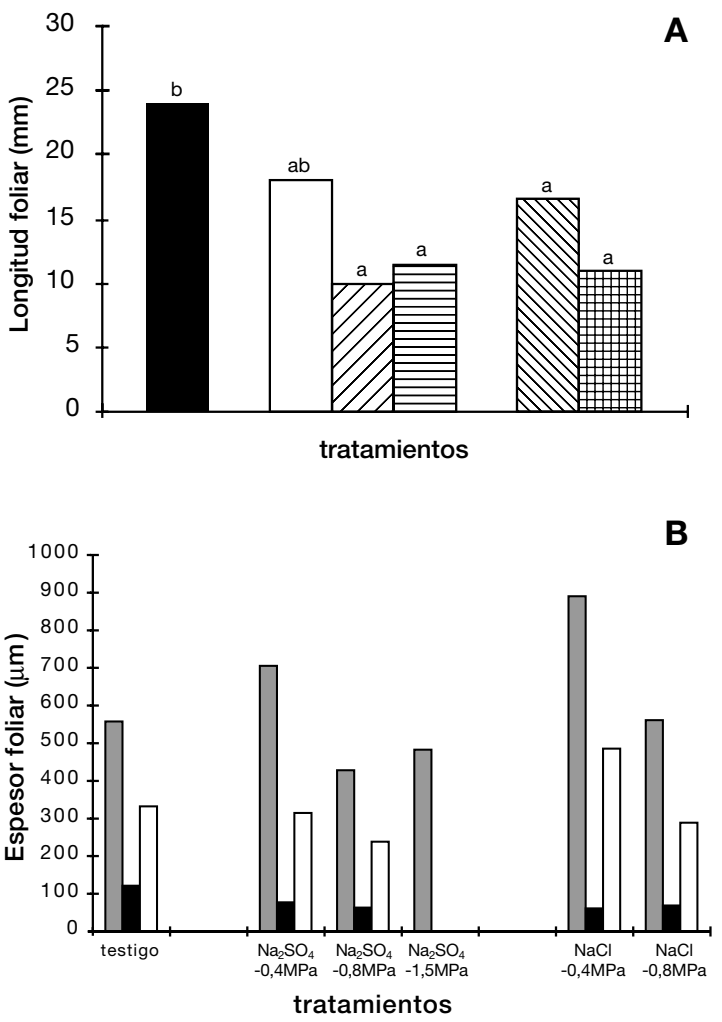

Figura 4. Efecto del medio salino: A. en la longitud ( $\square$ testigo; $\square \mathrm{Na}_{2} \mathrm{SO}_{4}-0,4 \mathrm{MPa} ; \square \mathrm{Na}_{2} \mathrm{SO}_{4}-0,8 \mathrm{MPa}$; 目 $\mathrm{Na}_{2} \mathrm{SO}_{4}-1,5 \mathrm{MPa}$;

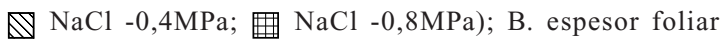
$\square$ espesor total, $\square$ espesor de la empalizada, $\square$ espesor del parénquima esponjoso). 
La capacidad para conducir agua disminuye en plantas expuestas a condiciones de salinidad, puesto que la alta concentración salina crea un hábitat de condiciones de sequía fisiológica (Ginzburg 1966, Fahn 1974). Dicha reducción en la capacidad conductora puede ser consecuencia de la disminución del tamaño de los vasos, efecto observado por Strogonov (1964) en plantas de algodón y tomate, y por Huang \& Redmann (1995a) en cebada silvestre. La disminución del espesor de la corteza de la raíz le permitiría al vegetal un mayor gradiente de potencial de agua entre la solución del suelo y el cilindro central, promoviendo así un mejor movimiento del agua disponible (Ginzburg 1966), efecto éste que parece manifestarse en las raíces de las plantas de T. absinthioides sometidas al sulfato de sodio.

La reducción de la altura de las plantas y aún la producción de achaparramiento es otro claro efecto de la incidencia de la salinidad en el crecimiento de las halófitas. Se observó en T. absinthioides una disminución significativa de la altura de las plantas, con entrenudos más cortos, como respuesta tanto a cloruro como a sulfato, a partir de un potencial osmótico de $-0,8 \mathrm{MPa}$ en el medio. El mismo efecto se cita para Atriplex halimus (Poljakoff-Mayber \& Gale 1975), sorgo (Mass et al. 1986) y algodón (Strogonov 1964) y cebada var. Harrington, que resultó más sensible a la salinidad producida por los sulfatos que a la producida por cloruros (Huang \& Redmann 1995b). En A. halimus la reducción fue tal que se produjeron plantas enanas (Poljakoff-Mayber \& Gale 1975). Estos autores le adjudican al achaparramiento un efecto beneficioso para el vegetal, pues da como resultado plantas con menor área transpiratoria y maduración y floración anticipadas, que le sirven como medio de escape al efecto perjudicial de las sales y debido probablemente a que durante el proceso de ajuste osmótico el alargamiento de los órganos se suspende para conservar el estado de turgencia (Shalhevet et al. 1995). Una respuesta rápida se observa en la disminución del crecimiento de las hojas (Munns \& Termaat 1986, Huang \& Redmann 1995b), carácter observado en $T$. absinthioides. Esta disminución del crecimiento sería el resultado de una falta de alargamiento en las células y no de una disminución de la división (Wignarajah et al. 1975, Voronin et al. 1995). Esta relación entre división y alargamiento explicaría el aumento del número de pelos en una sección conocida, con el aumento de la concentración de las sales. El parénquima en empalizada de las plantas de T. absinthoides tratadas con sales fue siempre más delgado que en las plantas control, como lo citan para Phaseolus Wignarajah et al. (1975) y Voronin et al. (1995) para las diferentes especies por ellos estudiadas. Los rangos de tolerancia para especies halófilas varían desde 0,07 M (Barbour 1970) a 1,11 M (St. Omer \& Schlesinger 1980) de cloruro de sodio. T. absinthioides crece entre 0,04 y $0,09 \mathrm{M}$, rango en el que su longitud total se ve disminuída, y muere en condiciones de salinidad en las que plantas halófilas muestran crecimiento óptimo (Greenway 1968). No se conocen límites de tolerancia de salinidad debida a sulfato de sodio, ya que el efecto de esta sal ha sido menos estudiado (Hayward \& Long 1941, Strogonov 1964, Huang \& Redmann 1995a). Ante esta sal fue más difícil identificar el mecanismo de adaptación utilizado. Se observa un gran aumento de la pubescencia en hoja a -0,4 MPa, conjuntamente con el aumento del número de lagunas en la corteza de la raíz y el aumento relativo de la corteza del tallo. Estos efectos son característicos de medios desecantes (Fahn 1974, Hansen et al. 1976). Quizás esta sal le produce mayor perjuicio al vegetal y como resultado un efecto haloxeromórfico. Para responder a la hipótesis conceptual planteada en la introducción general de este trabajo se comparan los resultados obtenidos en este ensayo con las curvas de crecimiento propuestas por Waisel (1972) para halófitas, glicófitas y semihalófitas, concluyendo que $T$. absinthioides no se comporta como una halófita verdadera, sino que muestra conductas que podrían enmarcarla entre las semihalófitas, puesto que crece en condiciones de baja salinidad, disminuyendo luego el crecimiento en forma lineal con el aumento de la concentración. La hipótesis específica planteada en la introducción de este artículo respecto a los mecanismos con que cuenta T. absinthioides para vivir en condiciones de salinidad, se comprueba con las conclusiones que surgen de estos resultados. T. absinthioides produce suculencia en los órganos vegetativos y achaparramiento cuando la sal involucrada es el $\mathrm{NaCl}$ y caracteres haloxeromórficos si la salinidad es por $\mathrm{Na}_{2} \mathrm{SO}_{4}$. 


\section{Referencias bibliográficas}

BARBOUR, M. 1970. Is any angiosperm an obligate halophyte? American Midland Naturalist 84: 105-120.

BLACK, R.F. 1958. Effect of sodium chloride on leaf succulence and area of Atriplex hastata L. Australian Journal of Botany 6:306-321.

FAHN, A. 1974. Plant anatomy. 2nd ed. Pergamon Press, Oxford. GINZBURG, C. 1966. Xerophytic structures in the roots of desert shrubs. Annals of Botany 30:403-418.

GREENWAY, H. 1968. Growth stimulation by high chloride concentration in halophytes. Israel Journal of Botany 17:169-177.

HANSEN, D.J., DAYANANDAN, P., KAUFMAN, P. \& BROTHERSON, J,.D. 1976. Ecological adaptations of saltmarsh grass, Distichlis spicata (Gramineae), and environmental factors affecting its growth and distribution. American Journal of Botany 63:635-650.

HAYWARD, H. E. \& LONG, E. M. 1941. Anatomical and physiological responses of the tomato to varying concentrations of sodium chloride, sodium sulphate and nutrient solutions. Botanical Gazzette 102:437-462.

HOAGLAND, D.R. \& ARNON, D.I. 1950. The culture method for growing plants without soil. California Agriculture Experimental Circle 347:1-32.

HUANG, J. \& REDMANN, R.E. 1995a. Salt tolerance of Hordeum and Brassica species during germination and early seedling growth. Canadian Journal of Plant Science 75:815-819.

HUANG, J. \& REDMANN, R.E. 1995b. Responses of growth, morphology, and anatomy to salinity and calcium supply in cultivated and wild barley. Canadian Journal of Botany 73:1859-1866

JENNINGS, D.H. 1968. Halophyte succulence and sodium in plants. A unified theory. New Phytologist 67:899-911.
JENNINGS, D.H. 1976. The effects of sodium chloride on higher plants. Biological Review 51:453-486.

MAAS, E.V., POOS, J.A. \& HOFFMAN, G.J. 1986. Salinity sensitivity of sorghum of three growth stages. Irrigation Science 7:1-11.

MUNNS, R. \& TERMAAT, A. 1986. Whole plant responses to salinity. Australian Journal of Plant Physiology 13:143-160.

POLJAKOFF-MAYBER, J. \& GALE, J. 1975. Plants in saline environments. Springer-Verlag, New York.

PURER, E.A. 1936. Studies of certain coastal sand dune plants of southern. California. Ecological Monographs 6:3-87.

SHALHEVET, J., MORRIS, G.K. \& SCHROEDER, B.P. 1995. Root and shoot growth responses to salinity in maize and soybean. Agronomy Journal 87:512-516

STATGRAPHICS. 1992. Version 6.0. Statistical Graphic System Corporation Inc.

ST. OMER, L. \& SCHLESINGER, W.H. 1980. Field and greenhouse investigations of the effect of increasing salt stress on the anatomy of Jaumea carnosa (Asteraceae), a salt marsh species. American Journal of Botany 67:455-465.

STROGONOV, B.P. 1964. Physiological basis of salt tolerance of plants (as affected by various types of salinity). Akad. Nauk. SSSR (Traducción del ruso, Isr. Progr. Sci. Transl., Jerusalem)

UPHOF, J.C.Th. 1941. Halophytes. Botanical Review 7:1-58

VORONIN, P.YU., MANZHULIN, A.V., MYASOEDOV, N.A. BALNOKIN, YU.V.\& TERENT'EVA, E.I. 1995. Morphological types and photosynthesis of $\mathrm{C}_{4}$ plant leaves under long-term soil salinity. Russian Journal of Plant Physiology 42:310-320

WAISEL, X. 1972. Biology of halophytes. Academic Press, New York.

WIGNARAJAH, K, JENNINGS, D.H. \& HANDLEY, J.F. 1975. The effect of salinity on growth of Phaseolus vulgaris. I. Anatomical changes in the first trifoliolate leaf. Annals of Botany 39:1029-1038. 